\title{
Microbial diversity and novel symbiotic Zetaproteobacteria species in Rimicaris exoculata
}

\author{
Marie-Anne Cambon-Bonavita \\ Johanne Aubé \\ Valérie Cueff-Gauchard \\ Julie Reveillaud
}

\section{Video Byte}

Keywords: niche partitioning, Rimicaris, metagenome-assembled genomes, Zetaproteobacteria, holobiont, hydrothermal vents, shrimp, deep-sea shrimp, symbiosis, Mid-Atlantic Ridge, shotgun metagenomics, symbiotic genomes, marine biology, marine microbes, microniche, chemosynthetic bacteria, epibionts, TAG vent field, Rainbow vent field, Snake Pit vent field, Microbiome

Posted Date: October 14th, 2021

DOI: https://doi.org/10.21203/rs.3.rs-968259/v1

License: (c) (1) This work is licensed under a Creative Commons Attribution 4.0 International License. Read Full License 


\section{Abstract}

Both free-living and symbiotic chemosynthetic microbes are critical to the health of deep-sea hydrothermal vent ecosystems. A complex symbiotic microbe community lives in the cephalothorax of the shrimp Rimicaris exoculata, which thrives in diverse vent environments. However, the taxonomic and functional diversity of this community is not fully characterized, and how the microbes contribute to the shrimp's success in vent colonization remains to be further studied. In a recent study, researchers used shotgun metagenomics to reconstruct and study symbiotic bacterial genomes in shrimp specimens from three vent fields with different geochemical characteristics. Forty-nine metagenome-assembled genomes were successfully reconstructed for shrimps from two of the fields. The communities were complex, and many microbes were shared between fields. However, behind an apparent functional redundancy, coexisting strains showed differences, highlighting niche partitioning between symbionts. In addition, some of the genomes belonged to potential novel species, including two in the class Zetaproteobacteria. This is the first report of symbiotic genomes for this class, and the sequences suggest that the two species share many, but not all, metabolic traits. Overall, the findings provide insights into how this remarkable shrimp may adapt to different vent environments and help fill knowledge gaps regarding these fascinating and difficult-to-study ecosystems on the sea floor. 